\title{
Physical Insights on Design and Modeling of Nanoscale FinFETs
}

\author{
J. G. Fossum, M. M. Chowdhury, V. P. Trivedi, T.-J. King*, Y.-K. Choi*, J. An**, and B. Yu** \\ University of Florida, Gainesville, FL 32611-6130, U.S.A. \\ *University of California, Berkeley, CA 94720 , U.S.A. \\ **AMD, Sunnyvale, CA 94088 , U.S.A.
}

\begin{abstract}
An array of measured device data, a numerical device simulator, and a process/physics-based compact model are used to gain new and important physical insights on nanoscale FinFETs with undoped thin-fin bodies. The insights, which include unavoidable/needed gate underlap, bias-dependent effective channel length, and non-ohmic fin-extension voltage drops, reveal the significance of gate positioning on, and source/drain doping profile in, the thin fin, and imply novel compact modeling that will be needed for optimal design of nonclassical CMOS circuits.
\end{abstract}

\section{Introduction}

As the scaling of classical (i.e., bulk-Si and PD/SOI) CMOS is approaching a palpable limit, nonclassical MOSFET structures having ultra-thin $\mathrm{Si}$ bodies which facilitate scaling, including multiple-gate devices [1], [2], are being earnestly examined. The FinFET [3], [4], a quasi-planar double-gate (DG) transistor, is attracting much attention now because it is relatively easy to fabricate. The gate structure (of length $L_{g}$ ) of the FinFET, illustrated in Fig. 1, is "wrapped" over a thin Si fin (of thickness $t_{\mathrm{Si}_{\mathrm{j}}}$ ), yielding two active vertical gates that are beneficially charge-coupled [5] if $t_{\mathrm{Si}}$ is sufficiently thin, a specification, relative to the effective channel length $\left(\mathrm{L}_{\mathrm{eff}}\right)$, which also ensures good control of short-channel effects (SCEs). The gate is necessarily separated from the source/ drain (S/D) contact regions by extensions of the thin Si fin, features which complicate the design and modeling of the device as we reveal herein.

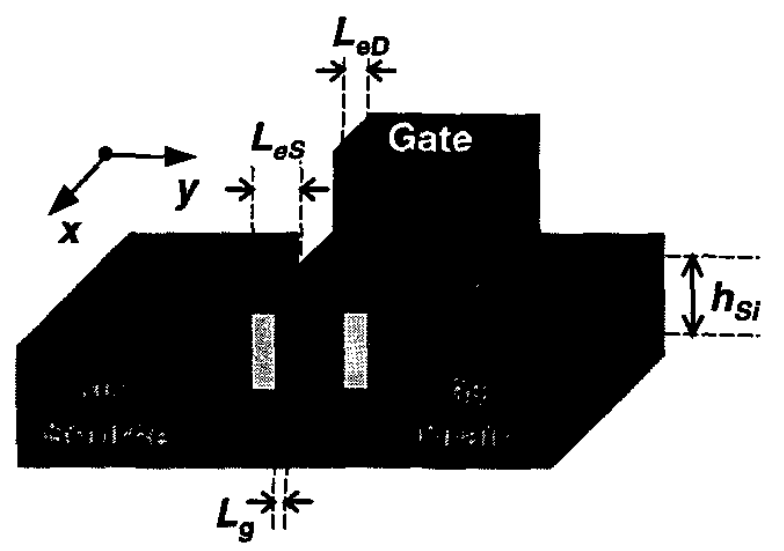

Fig. 1. The n-channel DG FinfET structure, with indications $\left(L_{e s}\right.$ and $\mathrm{L}_{\mathrm{ep}}$ ) of the undoped portions of the S/D fin extensions which are technologically unavoidable when the body of the nanoscale device is undoped. The gate for the nFinFETs studied herein [4] is $\mathrm{n}^{+}$ polysilicon, and the gate insulator is nitrided oxide with physical thickness $\mathrm{t}_{\mathrm{ox}}=1.7 \mathrm{~nm}$.

\section{Insights}

The height $\left(\mathrm{h}_{\mathrm{Si}}\right)$ of the $\mathrm{Si}$ fin, which is the effective gate width of the DG FinFET, is technologically limited to be not much larger than $t_{S i}$. Hence, the volume of the Si-channel region under the gate (i.e., $\mathrm{h}_{\mathrm{Si}} \mathrm{t}_{\mathrm{Si}} \mathrm{L}_{\mathrm{g}}$ ) of the nanoscale $\left(\mathrm{L}_{\mathrm{g}}<50 \mathrm{~nm}\right)$ FinFET is small, which, technologically, tends to preclude the use of body doping for threshold control. (Metal gates with tuned work functions will probably be needed.) With undoped bodies and the implied low inherent doping density, the small FinFET body/channel will likely be virtually void of any dopants (i.e., intrinsic), and, because of technological limitations, so will portions (of lengths $\mathrm{L}_{\mathrm{eS}}$ and $\mathrm{L}_{\mathrm{eD}}$ as indicated in Fig. 1) of the S/D extensions adjacent to the gate. In fact, such a gate-underlap structure could be required to get optimal DG CMOS circuit speeds when $L_{g}<25 \mathrm{~nm}[6]$. Note that $\mathrm{L}_{\mathrm{eS}}$ and $\mathrm{L}_{\mathrm{eD}}$ are defined by the S/D lateral doping profile in the thin-fin extensions.

Consider $\mathrm{L}_{\mathrm{eff}}$, which can be thought of as the length over which the gate modulates the channel, or S-D conductivity; it governs SCEs as well as channel current $\left(I_{c h}\right)$. For weak inversion, MEDICI [7] simulations of 2D cross-sections ( $x-y$ plane in Fig. 1) of nFinFETs show, in Figs. 2 and 3, that $\mathrm{L}_{\text {eff }}>$ $L_{g}$, i.e., that $V_{G S}$ modulates the electric potential $(\phi)$ and the electron density (n) in the undoped fin-extension regions as well as in the channel. This modulation is a result of gateinduced electrons moving into the extensions and modifying the electric field to effect a perturbed drift-diffusion detailed balance in accord with $I_{c h}$. The modulation is governed, via Poisson's equation, by the Debye length, $\mathrm{L}_{\mathrm{D}} \propto 1 / \sqrt{n}$. For weak inversion, $n$ is low and $L_{D}$ is relatively long (except near the S/D contact regions), implying

$$
L_{e f f(w e a k)} \cong L_{g}+L_{e S}+L_{e D},
$$

which is supported by the MEDICI results, including plots of $1 / \mathrm{I}_{\mathrm{ch}}$ vs. $\mathrm{L}_{\mathrm{g}}$ in Fig. 4. Further, as exemplified in Figs. 5-7, the long $\mathrm{L}_{\text {eff(weak) }}$ in (1) is corroborated by calibrations of our process/physics-based model UFDG [8] to FinFETs that were fabricated using the process described in [4], in which the body/channel is left undoped and the $S / D$ fin extensions are doped by $0^{\circ}$-tilt ion implantation with gate sidewall spacers, thereby yielding $\mathrm{L}_{\mathrm{eS}}, \mathrm{L}_{\mathrm{eD}}>0$. The noted $\mathrm{L}_{\mathrm{D}}(\mathbf{n})$ dependence on $\mathrm{V}_{\mathrm{GS}}$ means that $L_{\text {eff. }}$ is bias-dependent. For strong inversion, $n$ is high and $L_{D}$ is relatively short, suggesting that

$$
L_{\text {eff (strong) }} \equiv L_{g} \text {, }
$$

which is also consistent with the MEDICI simulation results (Figs. 2 and 3). The $\mathrm{L}_{\mathrm{eff}}\left(\mathrm{V}_{\mathrm{GS}}\right.$ ) dependence reflected by (1) and (2) intimates a possible novel performance advantage for FinfETs. The longer $L_{\text {eff }}$ in (1) implies lower $I_{\text {off }}$ and better controlled SCEs, as evidenced by the measured characteristics in Fig. 8 where $L_{g}=10 \mathrm{~nm}$ is significantly less than $L_{\text {eff(weak), }}$ 


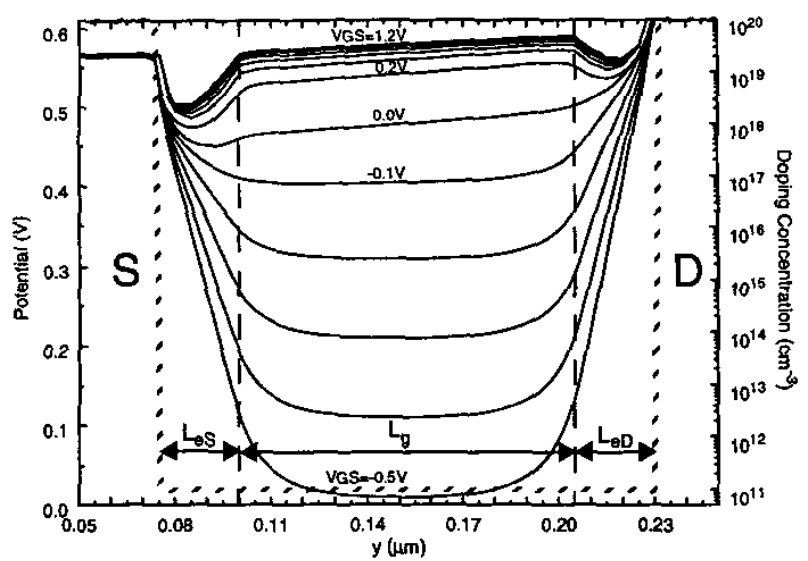

(a) $V_{D S}=0.1 \mathrm{~V}$

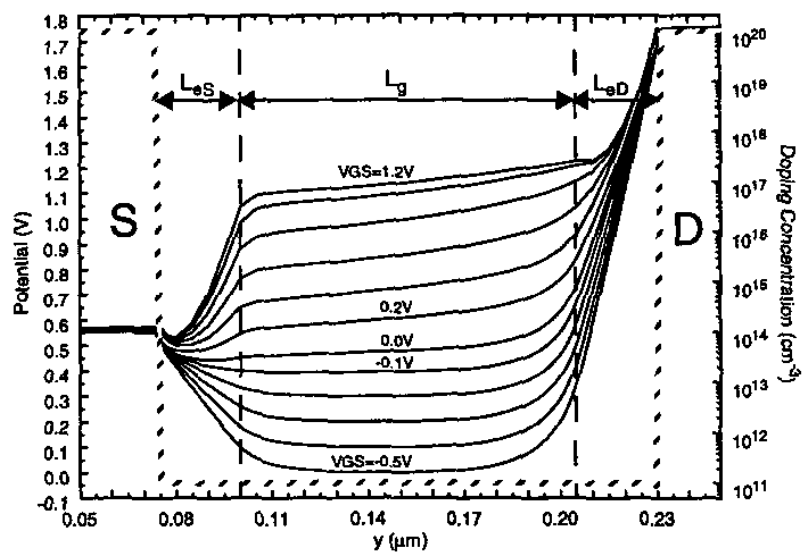

(b) $V_{D S}=1.2 \mathrm{~V}$

Fig. 2. MEDICI-predicted surface $\left(x=0, t_{S i}\right)$ potential variation between the $S$ and D contact regions of an $L_{g}=105 n m$ DG $n$ FinFET $\left(V_{t} \sim 0\right)$ for $V_{G S}$ varying between weak and strong inversion, and for (a) low and (b) high $V_{D S} ; t_{S i}=26 n m$. The entire S/D fin-extension regions $\left(\mathrm{L}_{\mathrm{es}}=\mathrm{L}_{\mathrm{eD}}=25 \mathrm{~nm}\right)$ were left undoped, as was the body/ channel. The S/D doping profile is abrupt as shown by the dotted curve.

while the shorter $\mathrm{L}_{\text {eff }}$ in (2) could mean higher $\mathrm{I}_{\text {on }}$ and lower gate capacitance, without parasitic components from the extensions, and no GIDL.

We note that the UFDG calibrations (Figs. 5 and 7) show the correlation between DIBL and $S$ with $\mathrm{L}_{\text {eff(weak) }}$ in (1). However, the UFDG calibrations to the measured data in strong inversion, with (2) enforced (Figs. 6 and 7), yield large, effective $\mathrm{S} / \mathrm{D}$ resistances that are asymmetric and $\mathrm{V}_{\mathrm{DS}^{-}}$ dependent, posing a potential problem. In accord with the MEDICI-predicted $n\left(V_{G S}, V_{D S}\right)$ in Fig. 3, these large resistances are actually indications of non-ohmic voltage drops $\left(\mathrm{V}_{\mathrm{eS}}\right.$ and $\left.\mathrm{V}_{\mathrm{eD}}\right)$ developed across both the $\mathrm{L}_{\mathrm{eS}}$ and $\mathrm{L}_{\mathrm{eD}}$ regions in support of $I_{c h}$, with $V_{e s}$ being most critical because it reduces the effective gate voltage and thereby tends to restrict the inversion charge density and $I_{o n} \cdot V_{e S}$ and $V_{e D}$ are non-ohmic because the conductivities of the extension regions are bias-dependent and the carrier transport is space-charge

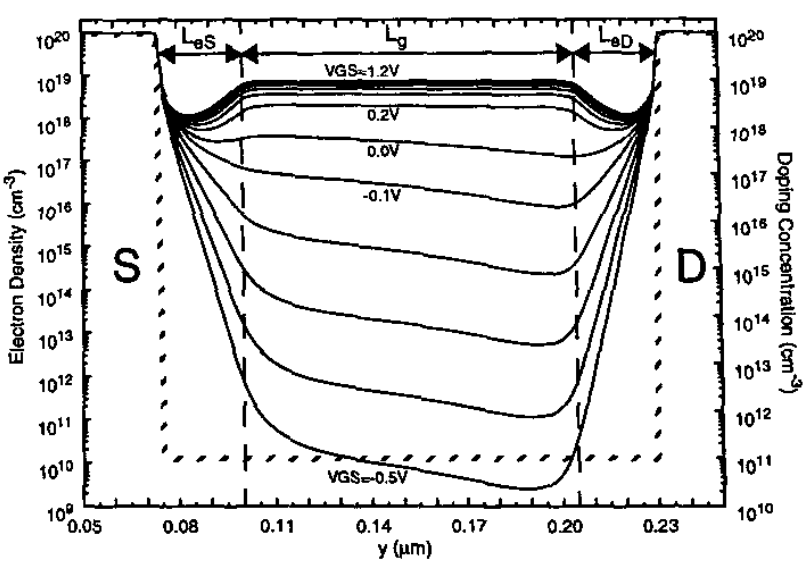

(a) $V_{D S}=0.1 \mathrm{~V}$

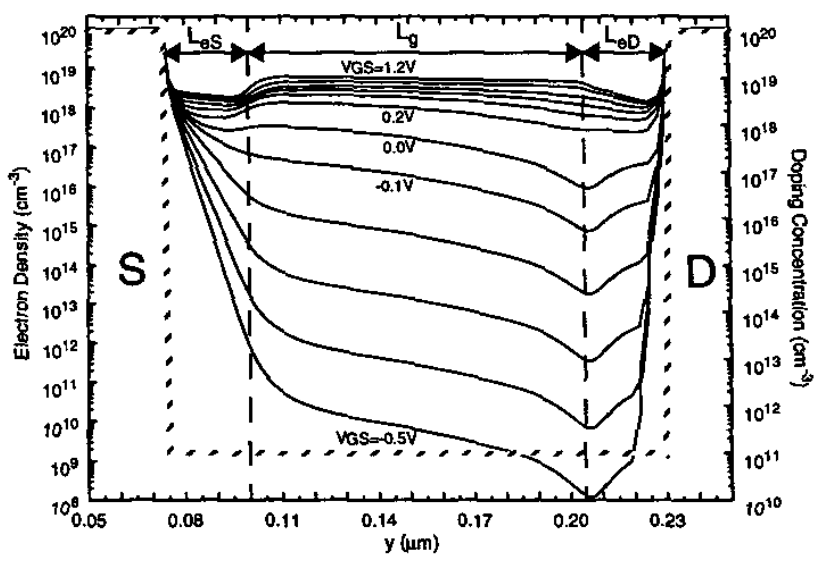

(b) $V_{D S}=1.2 \mathrm{~V}$

Fig. 3. MEDICI-predicted electron density (at $x=0, t_{S j}$ ) variation corresponding to the potential variation in the $\mathrm{L}_{\mathrm{g}}=105 \mathrm{~nm}$ DG nFinFET of Fig. 2.

limited, and, in fact, carrier velocity saturation in this transport can, as implied by the high electric fields $\left(E_{e}=-d \phi / d y\right)$ in Fig. 2(b), define $\mathrm{I}_{\mathrm{on}}$ if $\mathrm{L}_{\mathrm{es}}$ or $\mathrm{L}_{\mathrm{eD}}$ is too long.

Hence, any exploitation of performance advantage implied by (1) and (2) will demand control of $L_{e s}$ and $L_{e D}$, which, as defined by achievable S/D doping-density gradients in the thin-fin extensions and subject to avoiding punchthrough and/ or random-doping issues, will not be easy. (Thicker- $t_{s i}$ extensions would help.) Previous MEDICI-based studies [6] showed, in accord with the implied advantage, that nonzero $\mathrm{L}_{\mathrm{eS}}$ and $\mathrm{L}_{\mathrm{eD}}$ are optimal with regard to nanoscale DG CMOS speed, but no analysis of the underlying device physics was done. The dependences on $\mathrm{L}_{\mathrm{eS}}$ and $\mathrm{L}_{\mathrm{eD}}$ of the SCEs, $\mathrm{I}_{\mathrm{on}}$, and $\mathrm{I}_{\text {off }}$ are more complex than (1) and (2) imply. MEDICIpredicted SCEs shown in Fig. 9 for varying $\mathrm{L}_{\mathrm{eS}}$, but with $\left(\mathrm{L}_{\mathrm{eS}}\right.$ $+\mathrm{L}_{\mathrm{eD}}$ ) fixed in accord with FinFET technology [4], indicate that $\mathrm{L}_{\mathrm{eS}}=\mathrm{L}_{\mathrm{eD}}$ is the optimal configuration for SCE control, and that the SCEs are most severe when $\mathrm{L}_{\mathrm{eS}}$ or $\mathrm{L}_{\mathrm{eD}}$ is zero. Note that since $\mathrm{S}$ depends only on device geometry, the constant $S$ for nonzero $L_{e S}$ and $L_{e D}$ in Fig. 9 confirms (1) for 


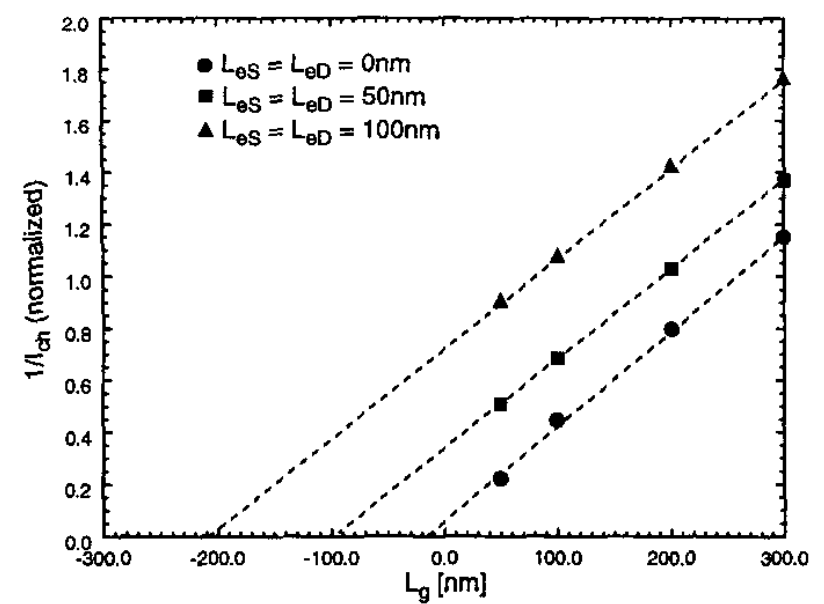

Fig. 4. MEDICI-predicted inverse weak-inversion channel current versus gate length of undoped DG nFinFETs with different S/D finextension (undoped) lengths; $t_{S i}=10 \mathrm{~nm}$. The $L_{g}$ intercepts of the linear extrapolations indicate $\mathrm{L}_{\mathrm{eff}}=0$ and confirm (1).

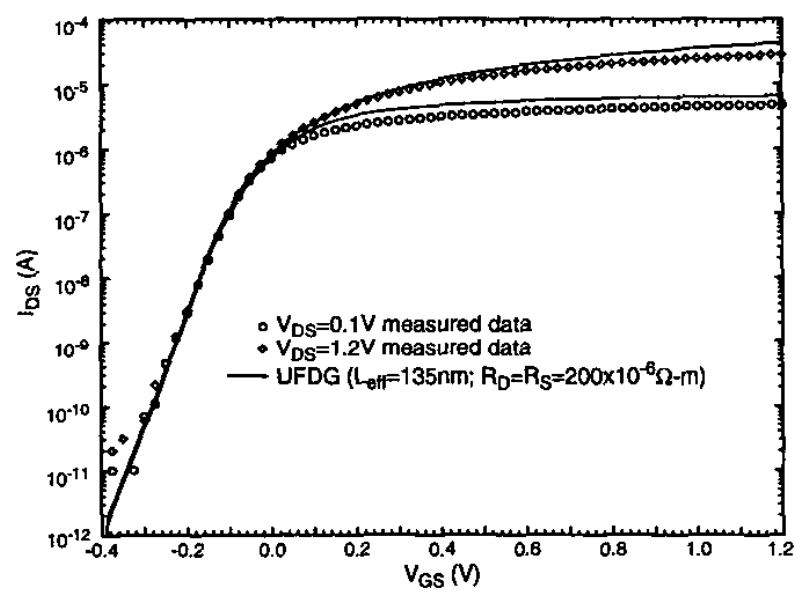

Fig. 5. Partial UFDG calibration to an $\mathrm{L}_{\mathrm{g}}=105 \mathrm{~nm}$ nFinFET; $\mathrm{t}_{\mathrm{Si}}=$ $26 \mathrm{~nm}$. With $\mathrm{L}_{\mathrm{eff}}=\mathrm{L}_{\mathrm{g}}+30 \mathrm{~nm}$ (as in (1)), the measured weakinversion $\mathrm{I}_{\mathrm{DS}}-\mathrm{V}_{\mathrm{GS}}$ characteristics are predicted well, but the stronginversion curves are not.

arbitrary gate position on the fin. However, note that DIBL and $V_{t}$ depend on the gate position, the explanation of which involves $\mathrm{L}_{\mathrm{D}}(\mathrm{n})$ as well as the $2 \mathrm{D}$ effects. Although $\mathrm{L}_{\mathrm{eS}}=\mathrm{L}_{\mathrm{eD}}$ is optimal for SCE control, generally $\mathrm{L}_{\mathrm{eS}} \neq \mathrm{L}_{\mathrm{eD}}$ due to technology limitations [4]. Further, we find, based on our derived physical insights and MEDICI-predicted $\mathrm{I}_{D S}\left(\mathrm{~V}_{G S}\right.$, $V_{D S}$ ) characteristics accounting for the non-ohmic voltage drops, that $\mathrm{L}_{\mathrm{eS}}<\mathrm{L}_{\mathrm{eD}}$ (i.e., the gate positioned closer to the source) is optimal with regard to maximum $I_{\text {on }} / I_{\text {off }}$.

\section{Compact Modeling}

The insights revealed in the previous section imply novel compact modeling needed for nonclassical CMOS circuit design, as well as optimal FinFET design criteria. The bias dependence of $L_{\text {eff }}$ is reflected by (1) and (2), but (1) must be

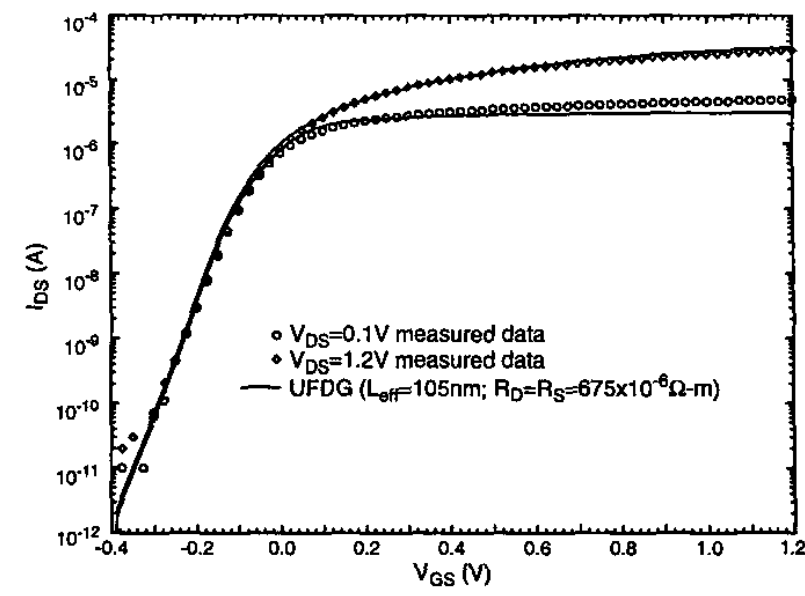

Fig. 6. Partial UFDG calibration to an $\mathrm{L}_{\mathrm{g}}=105 \mathrm{~nm} n$ FinFET; $\mathrm{t}_{\mathrm{Si}}=$ $26 \mathrm{~nm}$. With $\mathrm{L}_{\mathrm{eff}}=\mathrm{L}_{\mathrm{g}}$ (as in (2)) and very high, but constant $\mathrm{S} / \mathrm{D}$ series resistance, the measured high- $V_{D S}$ strong-inversion $I_{D S}-V_{G S}$ characteristic is predicted well, but the low- $V_{D S}$ and weakinversion curves are not.

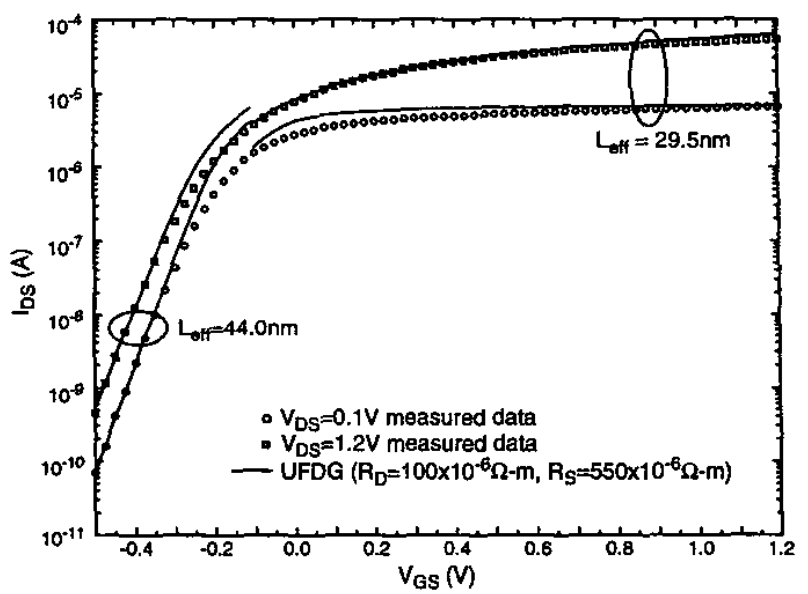

Fig. 7. Partial UFDG calibration to an $\mathrm{L}_{\mathrm{g}}=17.5 \mathrm{~nm}$ nFinFET; $\mathrm{t}_{\mathrm{Si}}=$ $17 \mathrm{~nm}$. With $\mathrm{L}_{\mathrm{eff}}=\mathrm{L}_{\mathrm{g}}+26.5 \mathrm{~nm}$, the measured weak-inversion $\mathrm{I}_{\mathrm{DS}^{-}}$ $\mathrm{V}_{\mathrm{GS}}$ characteristics are predicted well; with shorter $\mathrm{L}_{\text {eff }}=\mathrm{L}_{\mathrm{g}}+$ $12.0 \mathrm{~nm}\left(\sim 2 \mathrm{~L}_{\mathrm{D}}\right)$, and very high source series resistance but low drain resistance, the strong-inversion curves are predicted reasonably well. However, the bias-dependent $L_{e f f}$ and the non-ohmic $V_{e S}$ and $V_{e D}$ clearly must be modeled more physically.

refined (or tuned) based on the S/D lateral doping profile in the thin-fin extension, and on $\mathrm{L}_{\mathrm{D}}$ there as indicated in Figs. 2 and 3. For strong inversion, $L_{\text {eff }}$ in (2) will be a bit longer due to finite $L_{D}(n)$ near the channel, which suggests a secondary $V_{G S}$ dependence that may be significant for extremely short $\mathrm{L}_{\mathrm{g}}$ as implied in Fig. 7. Further, as implied in Fig. 9, $\mathrm{L}_{\mathrm{eff}}$ for $I_{c h}$ modeling could differ from that for SCEs.

Also, for strong inversion, $\mathrm{V}_{\mathrm{eS}}$ and $\mathrm{V}_{\mathrm{eD}}$ must be modeled. As can be inferred from Figs. 2(a), 3(a), 6, and 7, for low $V_{D S}$ (and $\mathrm{I}_{\mathrm{DS}}$ ), the $\mathrm{L}_{\mathrm{es}}$ and $\mathrm{L}_{\mathrm{eD}}$ regions can be modeled as constant resistances defined by carrier densities that have saturated with increased $V_{G S}$ in accord with decreased $L_{D}(n)$ relative to 


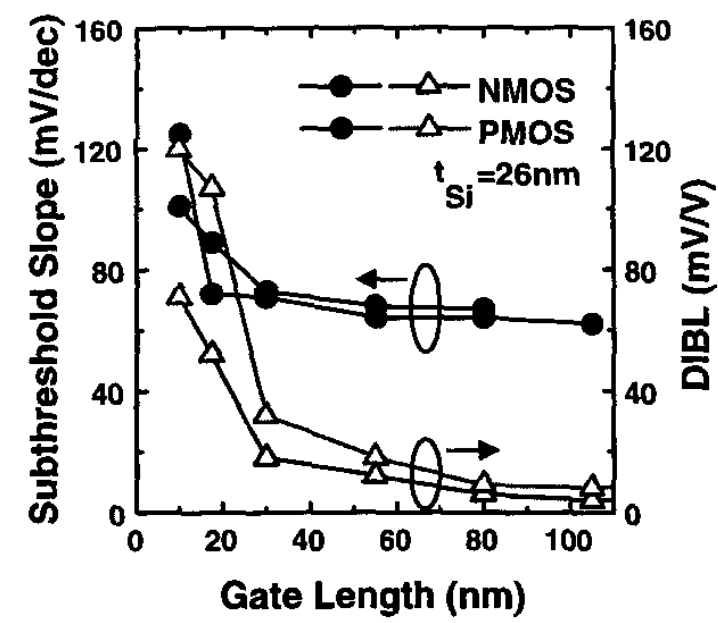

Fig. 8. Measured SCEs vs. $L_{\mathrm{g}}$ of CMOS FinFETs. The good control of DIBL and $S$ in the nanoscale devices is due to $L_{\text {eff }}>L_{g}$, as approximated by (1).

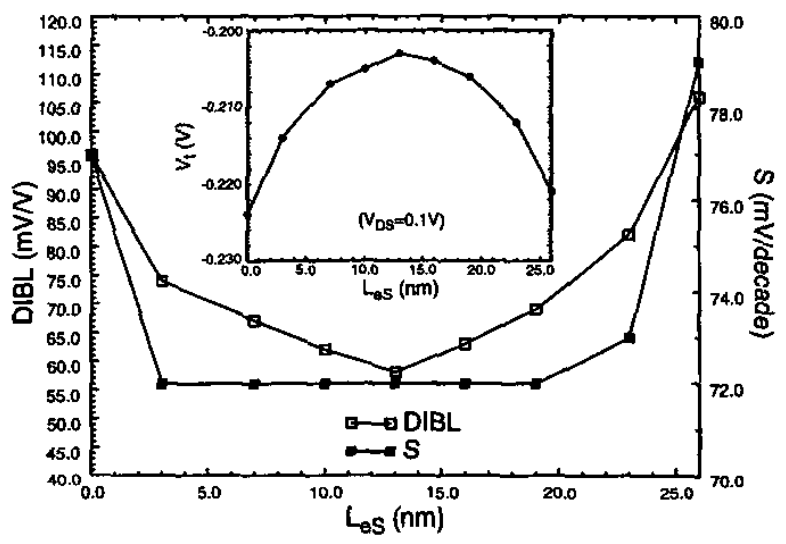

Fig. 9. MEDICI-predicted SCEs versus the source fin-extension length of $L_{g}=17.5 \mathrm{~nm}$ DG $n$ FinFETs for which $\left(\mathrm{L}_{\mathrm{es}}+\mathrm{L}_{\mathrm{eD}}\right)$ is fixed at $26 \mathrm{~nm} ; \mathrm{t}_{\mathrm{Si}}=12 \mathrm{~nm}$. The entire $\mathrm{S} / \mathrm{D}$ extensions and the body/channel were left undoped. The complex dependences on $L_{e s}$ and $L_{e D}$ are further reflected by the predicted linear-region threshold voltage $\left(V_{t}\right)$ shown in the inset.

$\mathrm{L}_{\mathrm{eS}}$ and $\mathrm{L}_{\mathrm{eD}}$, respectively. For high $\mathrm{V}_{\mathrm{DS}}$, Figs. 2(b) and 3(b) suggest that carrier-velocity saturation, with possible overshoot [9], in the $\mathrm{L}_{\mathrm{eS}}$ and $\mathrm{L}_{\mathrm{eD}}$ regions could define the saturation-region $I_{c h}$. To check this suggestion, we roughly calibrated MEDICI to the $\mathrm{L}_{\mathrm{g}}=17.5 \mathrm{~nm}$ nMOSFET of Fig. 7, and monitored the electron velocity variation across the device. Indeed, as shown in Fig. 10, velocity saturation and overshoot are predicted in the fin-extension regions. So, for a fixed $V_{G S}$, which defines $n$ in the regions as described above, the non-ohmic drops $V_{e S}$ and $V_{e D}$ will have to be modeled iteratively coupled to the channel-current analysis, similarly to the modeling of LDD voltage drops in high-voltage LDMOSTs [10]. The key expression for each region is

$$
I_{c h}=-h_{S i} Q_{n}\left(V_{G S}\right) v\left(E_{e}\right)
$$

where $Q_{n}$ is the integrated (over $t_{S i}$ ) inversion charge density

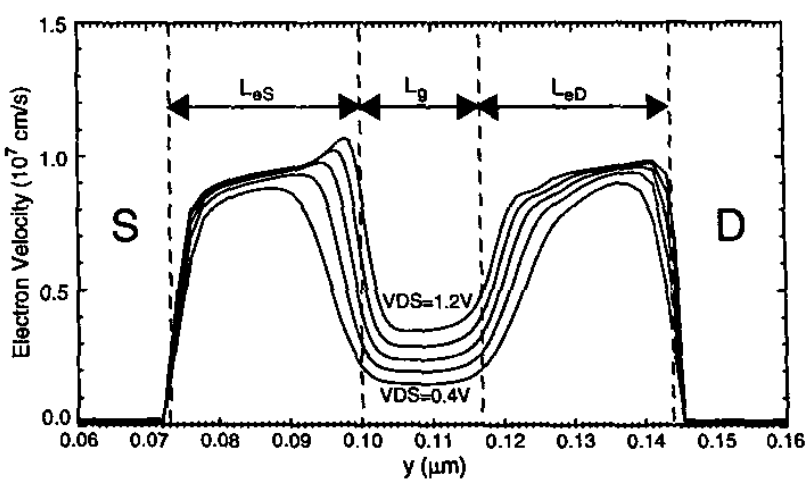

Fig. 10. MEDICI-predicted average electron velocity (at $x=0, t_{S i}$ ) variation between the $S$ and $D$ contact regions of an $L_{g}=17.5 \mathrm{~nm}$ DG $n$ FinFET, comparable to that of Fig. 7, for increasing $V_{D S} ; t_{S i}=$ $17 \mathrm{~nm}, \mathrm{t}_{\mathrm{ox}}=1.7 \mathrm{~nm}$. The entire $\mathrm{S} / \mathrm{D}$ fin-extension regions $\left(\mathrm{L}_{\mathrm{eS}}=\mathrm{L}_{\mathrm{eD}}\right.$ $=27 \mathrm{~nm}$ ) were left undoped, as was the body/channel. The S/D doping profile was assumed to be abrupt. With the energy-balance transport option on, MEDICl predicts velocity saturation, with some overshoot, in the fin-extension regions.

and $\mathrm{v}$ is the average carrier velocity, which saturates (at $\left.v_{\text {sat(eff) }}>v_{\text {sat }}[9]\right)$ with increasing $E_{e}$. The integrals of $E_{e}$ across $L_{e s}$ and $L_{e D}$ define the non-ohmic $V_{e s}$ and $V_{e D}$, respectively, which iteratively [10] redefine $I_{c h}$ in terms of $\left(\mathrm{V}_{\mathrm{DS}}-\mathrm{V}_{\mathrm{eS}}-\mathrm{V}_{\mathrm{eD}}\right)$.

\section{Summary}

While alignment issues [4] regarding gate positioning may complicate the design optimization of nanoscale FinFETs as suggested here, the physical insights revealed (i.e., the unavoidable/needed gate underlap, the bias-dependent $\mathrm{L}_{\mathrm{eff}}$, and the non-ohmic fin-extension voltage drops) will aid pragmatic optimal FinFET design, as well as the development of a complete physics-based compact model that will be needed for optimal designs of nonclassical CMOS circuits.

\section{Acknowledgments}

This work was supported in part by the Semiconductor Research Corporation and Motorola, Inc.

\section{References}

[1] R. Chau, et al., Ext. Abst. Internat. Conf. Solid State Devices \& Materials, pp. 68-69, Sept. 2002.

[2] J.-T. Park and J.-P. Colinge, IEEE Trans. Electron Devices, vol. 49 , Dec. 2002 , pp. 2222-2227.

[3] X. Huang, et al., IEEE IEDM Tech. Dig., pp. 67-70, Dec. 1999.

[4] B. Yu, et al., IEEE IEDM Tech. Dig., pp. 251-254, Dec. 2002.

[5] K. Kim and J. G. Fossum, IEEE Trans. Electron Devices, vol. 48, Feb. 2001, pp. 294-299.

[6] S. Balasubramanian, et al., Proc. Silicon Nanoelectronics Workshop, pp. 16-17, June 2003.

[7] MEDICI-4.0 User's Manual, Avant! Corp., Fremont, CA, 1999.

[8] UFDG-2.3 MOSFET Model User's Guide, SOI Group, Univ. Florida, Gainesville, FL, Apr. 2003.

[9] L. Ge, J. G. Fossum, and B. Liu, IEEE Trans. Electron Devices, vol. 48, Sept. 2001, pp. 2074-2080.

[10] Y.-S. Kim, J. G. Fossum, and R. K. Williams, IEEE Trans. Electron Devices, vol. 38, July 1991, pp. 1641-1649. 